\title{
ON THE INFORMATION-THEORETICAL ENTROPY FOR SOME QUANTUM OSCILLATORS
}

\author{
Dušan Popov ${ }^{1}$, Nicolina Pop ${ }^{1 *}$ and Simona Șimon ${ }^{2}$ \\ 1 "Politehnica" University of Timişoara, Department of Physical Foundation of Engineering, \\ 2 Vasile Pârvan Blvd., 300223 Timisoara,_Romania, E-mail: dusan_popov@yahoo.co.uk \\ *Corresponding author: E-mail: nicolina.pop@et.upt.ro \\ 2 "Politehnica" University of Timişoara, Faculty of Communication Sciences, 2 A Traian Lalescu St, 300223 \\ Timişoara, Romania
}

Article Info

Received: 24.03 .2013

Accepted: 08.04.2013

Keywords: Information-theoretical entropy, coherent state, Husimi's function.

PACS: 05.30.-d; 03.65.Fd;

42.50.Ar

\begin{abstract}
The information-theoretical entropy, also called the "classical" entropy, was introduced by Wehrl in terms of the Glauber coherent states (CSs) $\mid z>$, i.e. the CSs corresponding to the one-dimensional harmonic oscillator (HO-1D). In the present paper, we have focused our attention on the examination of the information-theoretical entropy, i.e. the Wehrl entropy, for both the pure and the mixed (thermal) states of some quantum oscillators.
\end{abstract}

\section{Introduction}

The information-theoretical entropy (or Wehrl entropy) was introduced by Wehrl [1] in terms of the Glauber coherent states (CSs) $\mid z>$ of the one-dimensional harmonic oscillator (HO-1D). In the present paper, we shall focus on the examination of the information-theoretical entropy for the two types of states: the pure and the mixed (thermal) states of some quantum oscillators: the one-dimensional harmonic oscillator (HO-1D) and the pseudoharmonic oscillator (PHO).

In terms of a general coherent state $\mid z, \lambda>$, the Wehrl entropy, defined via the Husimi's function $Q\left(|z|^{2}, \lambda\right)$, can be written as

$$
S_{W}=-\int d \mu(z, \lambda) Q\left(|z|^{2}, \lambda\right) \ln Q\left(|z|^{2}, \lambda\right)
$$


where $\lambda$ is a parameter which characterize the $\mathrm{CS}, d \mu(z ; \lambda)$ is the positive defined integration measure in the complex space of variable $z=|z| \exp (\mathrm{i} \varphi)$, with the corresponding weight function $h\left(|z|^{2} ; \lambda\right)$..

The general structure of a CS $\mid z, \lambda>$ is [2]

$$
\left|z ; \lambda>=\left[\mathcal{N}\left(|z|^{2} ; \lambda\right)\right]^{-\frac{1}{2}} \sum_{n=0}^{M} \frac{z^{n}}{\sqrt{\rho(n ; \lambda)}}\right| n ; \lambda>
$$

where $M \leq \infty$ and the structure constants $\rho(n ; \lambda)$ are positive quantities. The normalization function $\mathcal{N}(|z| ; \lambda)$ and the overlap of two CSs (where $\sigma$ is also the complex variable) are:

$$
\mathcal{N}\left(|z|^{2} ; \lambda\right) \equiv \sum_{n=0}^{M} \frac{\left(|z|^{2}\right)^{n}}{\rho(n ; \lambda)}<\infty . \quad<z ; \lambda \mid \sigma ; \lambda>=\frac{\mathcal{N}\left(z^{*} \sigma ; \lambda\right)}{\sqrt{\mathcal{N}\left(|z|^{2} ; \lambda\right) \mathcal{N}\left(|\sigma|^{2} ; \lambda\right)}}
$$

For the pure CSs the corresponding density operator is just the projector onto the complex space of these states:

$$
\rho^{(\text {pure })}\left(z, z^{*} ; \lambda\right)=|z, \lambda><z, \lambda|
$$

and the Husimi's function is $\left.Q_{n}^{(\text {pure })}\left(|z|^{2} ; \lambda\right)=|<n ; \lambda| z ; \lambda\right\rangle\left.\right|^{2}$.

For a mixed state, the associated density operator $\rho$ in a diagonal form with respect to the CSs is:

$$
\rho=\int d \mu(z, \lambda)\left|z, \lambda>P\left(|z|^{2}, \lambda\right)<z, \lambda\right|
$$

where $P\left(|z|^{2}, \lambda\right)$ is the normalized $P$ - quasi distribution function.

The $Q$-function or the Husimi's function is in fact the diagonal element of the density operator in the CSs representation and consequently it can be connected with the normalized $P$ quasi distribution function as follows:

$$
\begin{aligned}
& Q\left(|\sigma|^{2}, \lambda\right) \equiv<\sigma, \lambda|\rho| \sigma, \lambda>=\int d \mu(z, \lambda)|<\sigma, \lambda| z, \lambda>\left.\right|^{2} P\left(|z|^{2}, \lambda\right)= \\
& \quad=\frac{1}{\mathcal{N}\left(|\sigma|^{2} ; \lambda\right)} \int d \mu(z, \lambda) \frac{\mathcal{N}\left(\sigma^{*} z ; \lambda\right) \mathcal{N}\left(\sigma z^{*} ; \lambda\right)}{\mathcal{N}\left(|z|^{2} ; \lambda\right)} P\left(|z|^{2}, \lambda\right)
\end{aligned}
$$


so that the information-theoretical entropy $S_{W}$ can be calculated if we know the $P$ - quasi distribution function.

Our definition of the Husimi's $Q$-function is slightly different to other authors (see, e.g. [3]) due to the absence of the factor $\pi^{-1}$ from the integration measure. So, the difference between our information-theoretical entropy $S_{W}$ and that of [3], $S_{W}^{(\text {Mir })}$ is just the term $\ln \pi$, i.e.: $S_{W}^{(M i r)}=\ln \pi-S_{W}$

The aim of the present paper is to calculate the information-theoretical entropy $S_{W}$ by beginning on the Husimi's $Q$-function for pure and mixed (thermal) states for two kinds of quantum oscillators: HO-1D and PHO in the CSs representation $\mid z, \lambda>$. Due to the presence of $\ln Q\left(|z|^{2}, \lambda\right)$, some integrals appearing in the expression of $S_{W}$ are very hard if not impossible to be solved in a closed form. Consequently, in order to calculate the information-theoretical entropy, it must use some appropriate approximations.

\section{General theory}

\section{a) Pure States}

The density operator for the pure Fock states is $\rho_{n}^{(\text {pure })}=|n ; \lambda><n ; \lambda|$ and the Husimi's function in the representation of the Fock-vectors is

$$
Q_{n n^{\prime}}^{(\text {pure })}=<n^{\prime} ; \lambda\left|\rho_{n}^{(\text {pure })}\right| n^{\prime} ; \lambda>=\left|<n^{\prime} ; \lambda\right| n ; \lambda>\left.\right|^{2}=\delta_{n n^{\prime}}
$$

and the information-theoretical entropy of the pure Fock state in the Fock-vectors representation is zero: $S^{(\text {pure })}=0$. Consequently, the information-theoretical or Wehrl entropy in the case of a discrete variable coincides with the Shannon entropy so that "the Shannon entropy for any Fock state is equal to zero" [4].

In terms of the general CSs representation, the normalized Husimi's function can be written as follows:

$$
Q_{n}^{(\text {pure })}\left(|z|^{2} ; \lambda\right)=|<n ; \lambda| z ; \lambda>\left.\right|^{2}=\left[\mathcal{N}\left(|z|^{2} ; \lambda\right)\right]^{-1} \frac{\left(|z|^{2}\right)^{n}}{\rho(n ; \lambda)},
$$

so that the information-theoretical entropy becomes 


$$
S_{W}^{(\text {pure })}(n)=-\int d \mu(z, \lambda) Q_{n}^{(\text {pure })}\left(|z|^{2}, \lambda\right) \ln \left[Q_{n}^{(\text {pure })}\left(|z|^{2}, \lambda\right)\right]
$$

If we consider the pure general coherent states, the corresponding projector is $\rho_{z}^{(\text {pure })}=|z ; \lambda><z ; \lambda|$ and the Husimi's function in representation of the CSs $\mid \sigma ; \lambda>$ becomes

$$
Q^{(\text {pure })}(\sigma ; z ; \lambda) \equiv<\sigma ; \lambda\left|\rho_{z}^{(\text {pure })}\right| \sigma ; \lambda>=|<\sigma ; \lambda| z ; \lambda>\left.\right|^{2}=\frac{\mathcal{N}\left(\sigma^{*} z ; \lambda\right) \mathcal{N}\left(\sigma z^{*} ; \lambda\right)}{\mathcal{N}\left(|\sigma|^{2} ; \lambda\right) \mathcal{N}\left(|z|^{2} ; \lambda\right)}
$$

Consequently, the information-theoretical entropy for this case is

$$
\begin{gathered}
S_{W}^{(\text {pure })}(|\sigma|, \lambda)=-\int d \mu(z, \lambda) Q^{(\text {pure })}(\sigma, z ; \lambda) \ln \left[Q^{(\text {pure })}(\sigma, z ; \lambda)\right]= \\
=-\frac{1}{\mathcal{N}\left(|\sigma|^{2} ; \lambda\right)} \int d \mu(z, \lambda) \frac{\mathcal{N}\left(\sigma^{*} z ; \lambda\right) \mathcal{N}\left(\sigma z^{*} ; \lambda\right)}{\mathcal{N}\left(|z|^{2} ; \lambda\right)} \ln \left[\frac{\mathcal{N}\left(\sigma^{*} z ; \lambda\right) \mathcal{N}\left(\sigma z^{*} ; \lambda\right)}{\left.\mathcal{N}\left(|\sigma|^{2} ; \lambda\right) \mathcal{N}\left(|z|^{2} ; \lambda\right)\right]}\right.
\end{gathered}
$$

However, in these cases the integrals are complicated because of the presence of some special functions (e.g. hypergeometric and Bessel functions).

\section{b) Mixed States}

As a representative example of the quantum system in the mixed states we consider a quantum oscillator's ideal gas (of HO-1D or of PHO) in thermodynamic equilibrium with a thermostat at temperature $T$. For such quantum systems the energy spectrum is linear $E_{n}(\lambda)=a n+b$, with $a=a(\lambda), b=b(\lambda)$, and the mixed states are the thermal states characterized by the normalized density operator with the canonical probability distribution:

$$
\rho=\frac{1}{Z(\beta)} \sum_{n=0}^{\infty} e^{-\beta E_{n}}|n ; \lambda><n ; \lambda|=\frac{1}{\bar{n}+1} \sum_{n=0}^{\infty}\left(\frac{\bar{n}}{\bar{n}+1}\right)^{n}|n ; \lambda><n ; \lambda| .
$$

Here the quantity $\bar{n}=\left(e^{\beta a}-1\right)^{-1}$ is the mean number of photons (bosons) or Bose Einstein distribution function, i.e. the mean photon number of thermal (blackbody) radiation at thermal equilibrium at temperature $T$. 
The normalized Husimi's function in the representation of the Fock-vectors $\mid n ; \lambda>$ is expressed through the diagonal elements of the density operator for mixed (thermal) states:

$$
Q_{n^{\prime}}(\bar{n})=<n^{\prime} ; \lambda|\rho| n^{\prime} ; \lambda>\frac{1}{\bar{n}+1}\left(\frac{\bar{n}}{\bar{n}+1}\right)^{n^{\prime}}
$$

and the information-theoretical entropy for both cases (HO-1D and PHO) coincides with the von Neumann entropy:

$$
S_{W}(\bar{n}) \equiv-\operatorname{Tr}(\rho \ln \rho)=-\sum_{n^{\prime}=0}^{\infty} Q_{n^{\prime}}(\bar{n}) \ln Q_{n^{\prime}}(\bar{n})=\ln (\bar{n}+1)+\ln \left(\frac{\bar{n}+1}{\bar{n}}\right)^{\bar{n}} .
$$

As regarded the normalized Husimi's function for the case of the linear spectra (for which $n=0,1,2, \ldots, \infty)$ in the general CSs representation, this can be expressed as

$$
Q\left(|z|^{2}, \lambda\right) \equiv<z, \lambda|\rho| z, \lambda>=C(\bar{n}) \frac{\mathcal{N}\left(\frac{\bar{n}}{\bar{n}+1}|z|^{2} ; \lambda\right)}{\mathcal{N}\left(|z|^{2} ; \lambda\right)}
$$

where the constant $C(\bar{n})$ is different for HO-1D and for PHO.

Consequently, the information-theoretical entropy for the mixed states is then

$$
\begin{gathered}
S_{W}(\bar{n})=-\int d \mu(z, \lambda) Q\left(|z|^{2}, \lambda\right) \ln Q\left(|z|^{2}, \lambda\right)= \\
=-C(\bar{n}) \int d \mu(z, \lambda) \frac{\mathcal{N}\left(\frac{\bar{n}}{\bar{n}+1}|z|^{2} ; \lambda\right)}{\mathcal{N}\left(|z|^{2} ; \lambda\right)} \ln \left[\begin{array}{c}
\mathcal{N}\left(\frac{\bar{n}}{\bar{n}+1}|z|^{2} ; \lambda\right) \\
\mathcal{N}\left(|z|^{2} ; \lambda\right)
\end{array}\right]
\end{gathered}
$$

By particularizing the normalization constant $C(\bar{n})$ of the Husimi's function $Q\left(|z|^{2}, \lambda\right)$ and the normalization function $\mathcal{N}\left(|z|^{2} ; \lambda\right)$ of the CSs, we can directly compute the informationtheoretical entropy. 


\section{The HO-1D versus the PHO through the information-theoretical entropy}

The potential of the one-dimensional harmonic oscillator (HO-1D) with the mass $m$ and the angular frequency $\omega$ in the variable $x$ is

$$
V^{(H O)}(x)=\frac{m \omega^{2}}{2} x^{2}
$$

which allows the construction of three kinds of CSs defined in three manners: a) as the eigenvectors of the lowering operator $a ; b$ ) as the result of the action of the displacement operator $D(z) \equiv \exp \left(z a^{+}-z^{*} a\right)$ and $\left.\mathrm{c}\right)$ as the states which minimize the uncertainty relations $(\Delta x)_{z}^{2}(\Delta p)_{z}^{2}=\hbar / 2$. For HO-1D, all the three manners lead to the same canonical coherent state.

For the HO-1D, we have $\lambda=0$ and $M \rightarrow \infty$. Consequently, all the three definitions of CSs are equivalent in the sense that they lead to the same expression for the CSs, denoted by $\mid z>$. They have all minimal properties imposed to the CSs by Klauder's prescriptions [5]: a) continuity in the complex variable $z=|z| \exp (\mathrm{i} \varphi)$; b) normalization; c) completeness. So, we have:

$$
\left|z>=e^{-\frac{1}{2}|z|^{2}} \sum_{n=0}^{\infty} \frac{z^{n}}{\sqrt{n !}}\right| n>\quad ; \quad d \mu^{(H O)}(z)=\frac{d^{2} z}{\pi}=\frac{d \varphi}{2 \pi} d\left(|z|^{2}\right)
$$

Moreover, the pseudoharmonic oscillator (PHO) potential is a central field potential in the coordinate $r$ :

$$
V^{(P H O)}(r)=\frac{m \omega^{2}}{8} r_{0}^{2}\left(\frac{r}{r_{0}}-\frac{r_{0}}{r}\right)^{2}+\frac{\hbar^{2}}{2 m} J(J+1) \frac{1}{r^{2}}
$$

where $m$ is the reduced mass of the quantum system described by the PHO potential (e.g. the diatomic molecule), $r_{0}$ is the equilibrium distance, and $J$ is the rotational quantum number.

The quantum group associated with the PHO potential is $\mathrm{SU}(1,1)[6]$ and, consequently, three different kind of CSs can be built: a) the CSs of the Barut-Girardello kind (BG-CSs); b) the CSs of the Klauder-Perelomov kind (KP-CSs) and c) the CSs of the Gazeau-Klauder kind (GK-CSs). 


\subsection{The Information-Theoretical Entropy for Pure States}

Generally, for a pure Fock state $|n ; \lambda\rangle$, the Husimi's function in the general CSs representation is:

$$
Q_{n}^{(\text {pure })}\left(|z|^{2} ; \lambda\right) \equiv<z ; \lambda\left|\rho^{(\text {pure })}\right| z ; \lambda>=|<n ; \lambda| z ; \lambda>\left.\right|^{2}
$$

For the HO-1D, the Fock vectors are $|n ; \lambda=0>=| n>$; in the CSs representation, the Husimi's function is just the Poisson distribution with the parameter $|z|^{2}$ :

$$
Q_{n}^{(H O)}\left(|z|^{2}\right)=|<n| z>\left.\right|^{2}=e^{-|z|^{2}} \frac{\left(|z|^{2}\right)^{n}}{n !}
$$

and we obtain that the information-theoretical entropy is:

$$
S_{W}^{(H O)}(n)=-\int \frac{d^{2} z}{\pi} Q_{n}^{(H O)}\left(|z|^{2}\right) \ln \left[Q_{n}^{(H O)}\left(|z|^{2}\right)\right]=-\frac{1}{n !} \int_{0}^{\infty} d\left(|z|^{2}\right) e^{-|z|^{2}}\left(|z|^{2}\right)^{n} \ln \left[e^{-|z|^{2}} \frac{\left(|z|^{2}\right)^{n}}{n !}\right]
$$

If we denote $|z|^{2} \equiv x$, here appear an integral which can be solved by using an auxiliary integral:

$$
\begin{aligned}
I(n) & \equiv \int_{0}^{\infty} d x e^{-x} x^{n} \ln x=\lim _{\eta \rightarrow 0} \int_{0}^{\infty} d x e^{-x}\left[x^{n+\eta} \ln x\right]=\lim _{\eta \rightarrow 0} \int_{0}^{\infty} d x e^{-x} \frac{d}{d \eta}\left(x^{n+\eta}\right)= \\
& =\lim _{\eta \rightarrow 0} \frac{d}{d \eta} \int_{0}^{\infty} d x e^{-x} x^{n+\eta}=\lim _{\eta \rightarrow 0} \frac{d}{d \eta} \Gamma(n+\eta+1)=\Gamma(n+1) \psi(n+1)
\end{aligned}
$$

The final result contains Euler's digamma function $\psi(n+1)[3]$ :

$$
S_{W}^{(H O)}(n)=\ln n !+n+1-n \psi(n+1)
$$

For the PHO, three kinds of CSs exist.

a.The Barut-Girardello coherent states (BG-CSs) are defined as eigenvectors of the lowering operator $K_{-}$:

$$
\left|z ; k>_{B G}=\sqrt{\frac{|z|^{2 k-1}}{I_{2 k-1}(2|z|)}} \sum_{n=0}^{\infty} \frac{z^{n}}{\sqrt{n ! \Gamma(n+2 k)}}\right| n ; k>; d \mu^{(B G)}(z ; \lambda)=2 \frac{d^{2} z}{\pi} K_{2 k-1}(2|z|) I_{2 k-1}(2|z|)
$$


The Husimi's function for a pure Fock-state $\mid n ; k>$ in the representation of BG-CSs is [6]

$$
Q_{n}^{(B G)}\left(|z|^{2} ; k\right)=|<n ; k| z ; k>\left._{B G}\right|^{2}=\frac{1}{n ! \Gamma(n+2 k)} \frac{|z|^{2 n+2 k-1}}{I_{2 k-1}(2|z|)}
$$

which leads to the following expression for the information-theoretical entropy:

$$
S_{W}^{(B G)}(n)=2 \frac{1}{n ! \Gamma(n+2 k)}\left\{\ln [n ! \Gamma(n+2 k)] I_{1}^{(B G)}(n ; k)-(2 n+2 k-1) I_{2}^{(B G)}(n ; k)+I_{3}^{(B G)}(n ; k)\right\}
$$

The first integral can be solved exactly, the third integral can be solved only in an approximate way, and for the second one we need an auxiliary integral:

$$
\begin{gathered}
I_{1}^{(B G)}(n ; k) \equiv \int \frac{d^{2} z}{\pi} K_{2 k-1}(2|z|)|z|^{2 n+2 k-1}=\frac{1}{2} n ! \Gamma(n+2 k) \\
I_{2}^{(B G)}(n ; k) \equiv \int \frac{d^{2} z}{\pi} K_{2 k-1}(2|z|)|z|^{2 n+2 k-1} \ln |z|=\lim _{\eta \rightarrow 0} \frac{\partial}{\partial \eta} \int_{0}^{\infty} d(|z|) K_{2 k-1}(2|z|)|z|^{2 n+2 k+\eta}= \\
=\lim _{\eta \rightarrow 0} \frac{\partial}{\partial \eta}\left[\Gamma\left(n+2 k+\frac{\eta}{2}\right) \Gamma\left(n+1+\frac{\eta}{2}\right)\right]=\frac{1}{2} n ! \Gamma(n+2 k)[\Psi(n+2 k)+\Psi(n+1)]
\end{gathered}
$$

In the end, the following result yields:

$$
\begin{aligned}
S_{W}^{(B G)}(n) & =\ln n !+\ln \Gamma(n+2 k)-\frac{1}{2}(2 n+2 k-1)[\Psi(n+2 k)+\Psi(n+1)]+ \\
& +\frac{4}{n ! \Gamma(n+2 k)} \int_{0}^{\infty} d(|z|) K_{2 k-1}(2|z|)|z|^{2 n+2 k} \ln I_{2 k-1}(2|z|)
\end{aligned}
$$

b. The Klauder-Perelomov coherent states (KP-CSs) are defined by the action of the displaced operator $D[\alpha(z)] \equiv \exp \left(\alpha K_{+}-\alpha^{*} K_{-}\right)$on the ground (fiducial) state $\mid 0 ; k>$. Their development in the Fock-vectors basis is [8]:

$$
\left|z ; k>_{K P}=\left(1-|z|^{2}\right)^{k} \sum_{n=0}^{\infty} \frac{z^{n}}{\sqrt{\frac{n ! \Gamma(2 k)}{\Gamma(n+2 k)}}}\right| n ; k>\quad d \mu^{(K P)}(z ; k)=(2 k-1) \frac{d^{2} z}{\pi} \frac{1}{\left(1-|z|^{2}\right)^{2}} .
$$


The Husimi's function for a pure Fock-state $\mid n ; k>$ in the representation of KP-CSs is

$$
Q_{n}^{(K P)}\left(|z|^{2} ; k\right)=|<n ; k| z ; k>\left._{K P}\right|^{2}=\frac{\Gamma(n+2 k)}{n ! \Gamma(2 k)}\left(1-|z|^{2}\right)^{2 k}\left(|z|^{2}\right)^{n}
$$

which leads to the following expression for the information-theoretical entropy:

$$
S_{W}^{(K P)}(n)=-\frac{(2 k-1) \Gamma(n+2 k)}{n ! \Gamma(2 k)} \int \frac{d^{2} z}{\pi}\left(1-|z|^{2}\right)^{2 k-2}\left(|z|^{2}\right)^{n} \ln \left[\frac{\Gamma(n+2 k)}{n ! \Gamma(2 k)}\left(1-|z|^{2}\right)^{2 k}\left(|z|^{2}\right)^{n}\right]
$$

The first integral are solved by using Eq. (3.251.1) of the book [7] and the last two integrals by using an auxiliary integral:

$$
\begin{gathered}
I_{2}^{(K P)}(n) \equiv \int \frac{d^{2} z}{\pi}\left(1-|z|^{2}\right)^{2 k-2}\left(|z|^{2}\right)^{n} \ln \left(1-|z|^{2}\right)= \\
=\lim _{\eta \rightarrow 0} \frac{\partial}{\partial \eta} \int_{0}^{1} d\left(|z|^{2}\right)\left[\left(1-|z|^{2}\right)^{2 k-2+\eta}\right]\left(|z|^{2}\right)^{n}=n ! \frac{\Gamma(2 k-1)}{\Gamma(n+2 k)}[\Psi(2 k-1)-\Psi(n+2 k)] \\
I_{3}^{(K P)}(n) \equiv \int \frac{d^{2} z}{\pi}\left(1-|z|^{2}\right)^{2 k-2}\left(|z|^{2}\right)^{n} \ln \left(|z|^{2}\right)=\lim _{\eta \rightarrow 0} \frac{\partial}{\partial \eta} \int_{0}^{1} d\left(|z|^{2}\right)\left[\left(|z|^{2}\right)^{n+\eta}\right]\left(1-|z|^{2}\right)^{2 k-2}= \\
=\lim _{\eta \rightarrow 0} \frac{\partial}{\partial \eta} \frac{\Gamma(n+1+\eta) \Gamma(2 k-1)}{\Gamma(n+2 k+\eta)}=n ! \frac{\Gamma(2 k-1)}{\Gamma(n+2 k)}[\Psi(n+1)-\Psi(n+2 k)]
\end{gathered}
$$

Finally, we obtain:

$$
S_{W}^{(K P)}(n)=\ln n !+\ln (2 k)_{n}+2 k \Psi(2 k-1)+n \Psi(n+1)-(n+2 k) \Psi(n+2 k)
$$

where $(a)_{n}=\Gamma(a+n) / \Gamma(a)$ are the Pochhammer symbols.

c. The Gazeau-Klauder coherent states (GK-CSs) have been built by performing some modifications in the usual definition of the CSs [9]: the parametrization of the usual CSs in a single complex number $z$ is extended by replacing $z$ by two independent real numbers $J \geq 0$ and $-\infty<\gamma<+\infty$, namely $z=\sqrt{J} \exp (-\mathrm{i} \varphi)$ and obtaining the CSs $|J ; \gamma\rangle$. In a previous paper [10] we have concretely obtained the GK-CSs for the PHO as:

$$
\left|J ; \gamma>=\frac{1}{\sqrt{{ }_{1} F_{1}(1 ; k+1 ; J / 2)}} \sum_{n=0}^{\infty} \frac{(\sqrt{J / 2})^{n} e^{-\mathrm{i} \gamma e_{n}}}{\sqrt{(k+1)_{n}}}\right| n ; k>
$$


where $e_{n}=2(n+k)$ are the dimensionless energy eigenvalues of the PHO, ${ }_{1} F_{1}(1 ; k+1 ; x)$ is the confluent hypergeometric function. The integration measure for GK-CSs is

$$
d \mu^{(G K)}(J ; \gamma)=\frac{1}{\Gamma(k+1)} \frac{d \gamma}{2 \pi} d(J / 2)_{1} F_{1}(1 ; k+1 ; J / 2)(J / 2)^{k} \exp (-J / 2)
$$

The Husimi's function for a pure Fock-state $\mid n ; k>$ in the representation of GK-CSs is defined as

$$
Q_{n}^{(G K)}(J ; \gamma)=|<n ; k| J ; \gamma>\left._{K P}\right|^{2}=\frac{1}{{ }_{1} F_{1}(1 ; k+1 ; J / 2)} \frac{(J / 2)^{n}}{(k+1)_{n}}
$$

Consequently, the following expression for the information-theoretical entropy becomes:

$$
\begin{aligned}
& S_{W}^{(G K)}(n)=-\int d^{(G K)}(z ; k) Q_{n}^{(G K)}\left(|z|^{2} ; k\right) \ln \left[Q_{n}^{(G K)}\left(|z|^{2} ; k\right)\right]=I_{1}^{(G K)}(n)+I_{2}^{(G K)}(n)+I_{3}^{(G K)}(n) \\
& I_{1}^{(G K)}(n) \equiv-\frac{1}{\Gamma(k+1)} \ln (k+1)_{n} \int_{-\pi}^{\pi} \frac{d \gamma}{2 \pi} \int_{0}^{\infty} d(J / 2)(J / 2)^{n+k} \exp (-J / 2)=-(k+1)_{n} \ln (k+1)_{n} \\
& I_{2}^{(G K)}(n) \equiv n \frac{1}{\Gamma(k+1)} \int_{-\pi}^{\pi} \frac{d \gamma}{2 \pi} \int_{0}^{\infty} d(J / 2)(J / 2)^{n+k} \exp (-J / 2) \ln (J / 2)=n(k+1)_{n} \Psi(n+k+1)
\end{aligned}
$$

$$
I_{3}^{(G K)}(n) \equiv-\frac{1}{\Gamma(k+1)} \int_{-\pi}^{\pi} \frac{d \gamma}{2 \pi} \int_{0}^{\infty} d(J / 2)(J / 2)^{n+k} \exp (-J / 2) \ln _{1} F_{1}(1 ; k+1 ; J / 2)
$$

Then, the final result is

$$
S_{W}^{(G K)}(n)=-(k+1)_{n} \ln (k+1)_{n}+n(k+1)_{n} \Psi(n+k+1)+I_{3}^{(G K)}(n)
$$

where the last integral can be solved only in a certain approximation.

Generally, for a mixed (thermal) state for HO-1D we have $\mathcal{N}\left(|z|^{2}\right)=\exp \left(|z|^{2}\right)$ and $C(\bar{n})=(\bar{n}+1)^{-1}$ then, we obtain for the information-theoretical entropy [3]: $S_{W}^{(H O)}(\bar{n})=1+\ln (\bar{n}+1)$

\subsection{The Information-Theoretical Entropy for Mixed (Thermal) States}

In the case of the mixed (thermal) state for $\mathrm{BG}-\mathrm{CSS}$ of $\mathrm{PHO}$ we have 


$$
\mathcal{N}^{(B G)}\left(|z|^{2} ; k\right)=|z|^{-2 k+1} I_{2 k-1}(2|z|) \quad ; \quad C^{(B G)}(\bar{n})=(\bar{n}+1)^{-1}
$$

so that Eq. (2.15), with the help of integral (6.575.5) from the book [7], leads to

$$
S_{W}^{(B G)}(\bar{n})=\ln (\bar{n}+1)+\frac{1}{2}(2 k-1) \ln \frac{\bar{n}}{\bar{n}+1}-\frac{1}{\bar{n}+1}\left(\sqrt{\frac{\bar{n}+1}{\bar{n}}}\right)^{2 k-1}\left[I_{2}^{(B G)}(\bar{n})-I_{3}^{(B G)}(\bar{n})\right]
$$

where $I_{2,3}^{(B G)}(\bar{n})$ are integrals of the following manner

$$
I_{2,3}^{(B G)}(\bar{n})=2 \int_{0}^{\infty} d(|z|)|z| K_{2 k-1}(2|z|) I_{2 k-1}\left(2 \sqrt{\frac{\bar{n}}{\bar{n}+1}}|z|\right) \ln I_{2 k-1}(2 A|z|)
$$

and where $A=\sqrt{\bar{n} /(\bar{n}+1)}$ for $I_{2}^{(B G)}(\bar{n})$ and $A=1$ for $I_{3}^{(B G)}(\bar{n})$. Due to the existing logarithm function, these integrals can be solved only approximately.

In the case of the mixed (thermal) state for KP-CSs of PHO we have

$$
\mathcal{N}^{(K P)}\left(|z|^{2} ; k\right)=\left(1-|z|^{2}\right)^{-2 k} ; \quad C^{(K P)}(\bar{n})=(\bar{n}+1)^{-1}
$$

By applying again Eq. (2.15) and (3.197.3) from [7], the following result yields:

$$
S_{W}^{(K P)}(\bar{n})=\ln (\bar{n}+1)-(\bar{n}+1)^{-1}(2 k-1)\left[I_{2}^{(K P)}(\bar{n})-I_{3}^{(K P)}(\bar{n})\right]
$$

The appearing integrals $I_{2,3}^{(K P)}(\bar{n})$ are of the following kind:

$$
I_{2,3}^{(K P)}(\bar{n})=\int_{0}^{1} d x(1-x)^{2 k-2}(1-C x)^{-2 k} \ln (1-D x)^{2 k}
$$

with $C=\bar{n}(\bar{n}+1)^{-1}$ and $D=1$ for $I_{2}^{(K P)}(\bar{n})$ and $D=\bar{n}(\bar{n}+1)^{-1}$ for $I_{3}^{(K P)}(\bar{n})$. These integrals can be solved by appealing to the auxiliary integrals, e.g.:

$$
\begin{gathered}
I_{2}^{(K P)}(\bar{n})=\int_{0}^{1} d x(1-x)^{2 k-2}\left(1-\frac{\bar{n}}{\bar{n}+1} x\right)^{-2 k} \ln (1-x)^{2 k}= \\
=2 k \lim _{\eta \rightarrow 0} \frac{\partial}{\partial \eta} \int_{0}^{1} d x(1-x)^{2 k-2+\eta}\left(1-\frac{\bar{n}}{\bar{n}+1} x\right)^{-2 k}=2 k \lim _{\eta \rightarrow 0} \frac{\partial}{\partial \eta}\left[\frac{1}{2 k-1+\eta}{ }_{2} F_{1}\left(2 k, 1 ; 2 k+\eta ; \frac{\bar{n}}{\bar{n}+1}\right)\right]
\end{gathered}
$$

where we have used the integral (3.197.3) from [7]. For the differentiation we have used the following rules: 


$$
\frac{\partial}{\partial \eta}\left[{ }_{2} F_{1}(a, b ; c ; C)\right]=-\sum_{n=0}^{\infty} \frac{(a)_{n}(b)_{n}}{(c)_{n}} \frac{\partial c}{\partial \eta}[\Psi(c)-\Psi(c+n)] \frac{C^{n}}{n !}
$$

for solving the integrals $I_{2,3}^{(K P)}(\bar{n})$. Finally, the information-theoretical entropy for the case of the thermal states of $\mathrm{PHO}$ in the KP-CSs representation is

$$
S_{W}^{(K P)}(\bar{n})=1+\ln (\bar{n}+1)+(2 k-1)^{-1}
$$

In the case of the mixed (thermal) state for $\mathbf{G K}-\mathbf{C S S}$ of $\boldsymbol{P H O}$ we have [10]

$$
\mathcal{N}^{(G K)}(J)=\left[{ }_{1} F_{1}(1 ; k+1 ; J / 2)\right]^{-1} ; \quad C^{(G K)}(\bar{n})=(\bar{n}+1)^{-1}
$$

and we lead to the following final expression for the information-theoretical entropy, where $x \equiv J / 2$ :

$S_{W}^{(G K)}(\bar{n})=-\int d^{(G K)}(J ; \gamma) Q^{(G K)}(J ; \bar{n}) \ln \left[Q^{(G K)}(J ; \bar{n})\right]=\ln (\bar{n}+1)+I_{2}^{(G K)}(\bar{n} ; k)-I_{3}^{(G K)}(\bar{n} ; k)$

The final result is obtained when by using the integrals of the following kind:

$$
I_{2,3}^{(G K)}(\bar{n} ; k)=-\frac{1}{(\bar{n}+1) \Gamma(k+1)} \int_{0}^{\infty} d x x^{k} \exp (-x)_{1} F_{1}(1 ; k+1 ; A x) \ln _{1} F_{1}(1 ; k+1 ; B x)
$$

and where $A=\bar{n}(\bar{n}+1)^{-1}$ and $B=\bar{n}(\bar{n}+1)^{-1}$ for $I_{2}^{(G K)}(\bar{n} ; k)$ and $B=1$ for $I_{2}^{(G K)}(\bar{n} ; k)$.

Of course, these two integrals cannot be solved exactly, only approximately or by computational methods.

\section{Concluding remarks}

One of the most fundamental concepts of quantum mechanics, with applications in many branches of physics (quantum optics, quantum information, atomic and molecular physics and so on) is the quantum entropy, particularly, the Wehrl entropy. The Wehrl entropy is in fact the classical informational-entropy associated with a quantum system, in terms (or in representation) of coherent states [11].

Based on the definition of the informational-theoretic entropy in terms of the Husimi $Q$ function, in the paper we have deduced and examined the expressions of this kind of entropy for pure as well as mixed (thermal states) for two kinds of quantum systems: the quantum ideal gas 
of the one-dimensional harmonic oscillators (HO-1D) and the pseudoharmonic oscillators (PHO), respectively, at canonical equilibrium at temperature $T$ with the environment. We have calculated the informational-theoretic entropy $S_{W}$ for some common optical fields, in particular, Fock states and coherent states for $\mathrm{HO}-1 \mathrm{D}$ and for three kinds of states of PHO: BarutGirardello, Klauder-Perelomov and Gazeau-Klauder.

Due to the presence of the terms containing $\ln Q\left(|z|^{2} ; \lambda\right)$ in the expression of the entropy $S_{W}$, which implies some special functions (hypergeometric or Bessel functions), some integrals are impossible to be solved in a close form. Consequently, in order to calculate them, we need to use either some approximations of the special functions or the computational methods. One can observe that, on the one hand, all the expressions of the informationaltheoretic entropy for the pure states in the representation of the coherent states strongly depend on the energy quantum number $n$ through the term $\ln n$ !. On the other hand, all the expressions

of the informational-theoretic entropy for the thermal states contain the term $\ln (\bar{n}+1)$ because all the $Q$-functions have the same normalization constant $(\bar{n}+1)^{-1}$ which shows the strong dependence on the equilibrium temperature $T$.

\section{References}

[1] A. Wehrl, Rep. Math. Phys. 16 (1979) 353.

[2] D. Popov, Density matrix - General properties and applications in physics of many particle systems, Editura Politehnica, Timisoara, 2004 (in Romanian)

[3] A. Miranowicz, H. Matsueda, M. R. B. Wahiddin, J. Phys. A: Math. Gen. 33 (2000) 5159.

[4] V. P. Belawkin, O. Hirota, R. L. Hudson, Quantum Communications and Measurement, Plenum Press, New York (1995).

[5] J. R. Klauder, J. Math. Phys. 4 (1963) 1055.

[6] D. Popov, J. Phys. A : Math. Gen. 34 (2001) 5283.

[7] I. S. Gradshteyn, I. M. Ryshik, Table of Integrals, Series and Products, Seventh ed., Academic Press, Amsterdam (2007).

[8] D. Popov, D. M. Davidovic, D. Arsenovic, V. Sajfert, Acta Phys. Slovaca 56 (2006) 445.

[9] J. -P. Gazeau, J. R. Klauder, J. Phys. A : Math. Gen. 32 (1999) 123.

[10] D. Popov, V. Sajfert, I. Zaharie, Physica A 387 (2008) 4459.

[11] A. Anderson, J. J. Halliwell, Phys. Rev. D 48 (1993) 2753. 